\title{
Experimental Investigation of Air Dehumidification and Regeneration Operations Using Packed Bed of Silica Gel Particles
}

\author{
Ahlem Zouaoui *, Leila Zili-Ghedira and Sassi Ben Nasrallah \\ Energy and Thermal Systems Laboratory, National Engineering School of Monastir, University of \\ Monastir, Avenue Ibn El Jazzar, 5019 Monastir, Tunisia \\ Email: zouaoui_ahlem@yahoo.fr
}

\begin{abstract}
Energy consumption is increasing gradually in the world. It is due to the growth of air conditioning need in response to higher summer temperatures. This causes an increase in electricity demand. Solar cooling is a relatively new, reliable and clean application of proven refrigeration technology able to improve comfort conditions, limit the impact on the environment and save energy. The use of "desiccant cooling" technique is particularly interesting because it avoids the refrigerants use that have a negative effect on the ozone layer (CFC, HCFC, HFC ...). The main element in a desiccant cooling system is the desiccator. In this study, an experimental test bed was made in the Energy and Thermal Systems Laboratory "LESTE" at National Engineering School of Monastir, Tunisia. This bench is designed to examine the optimal conditions used (air flow rates effect) for a cylindrical desiccator, filled with silica gel grains, during both adsorption and desorption phases. For this aim, measurements of temperature, humidity and velocity at the inlet and outlet of the desiccator are done.
\end{abstract}

Keywords: Experimental, Dehumidification, Regeneration.

\section{INTRODUCTION}

The humidification and dehumidification processes of air are important operation in various industrial applications. Also, desiccant cooling system is alternate suitable option against conventional system in humid climatic condition for thermal comfort. The basic constituent of the desiccant cooling system is the desiccant bed.

Solid desiccants such as silica gel, zeolites, activated alumina, or hygroscopic salts are generally used to dehumidify moist air, and in such cases, the desiccant is continuously reactivated (regenerated). These adsorbents remove moisture from the air and then the adsorbed water is released in order to regenerate them using thermal energy such us electric heat, solar energy and waste heat. The heat and mass transfer characteristics of the bed have no doubt significant effects on the performance of the cooling system, and should therefore be adequately considered.

Silica gel can be desorbed at low temperature which makes it useful for use with solar energy [1]. An investigation on simultaneous dehumidification of silica gel showed that silica gel transfers about $30 \%$ more water per unit dry mass than activated alumina [2].

Theoretical and experimental study on the transient adsorption characteristics of vertical packed porous bed was studied by Hamed [3]. The performance of adsorption column of porous bed impregnated with calcium chloride as the desiccant is investigated. The experimental part of this study is based on the application of an inert material (porous granules of burned clay) as desiccant carrier.

Kim et al. [4] reported bench scale tests of a laminar flow silica gel packing element for solar air conditioning applications. The packing is based on a coated sheet concept with a single layer of $0.25 \mathrm{~mm}$ silica gel particles lining narrow passages of width $1.46-3.75 \mathrm{~mm}$. Adsorption and desorption tests were performed for fixed inlet conditions and a uniform initial bed condition.

A.E. Kabeel [5] studied theoretically and experimentally the effect of design and operating parameters on the performance of a multilayer silica gel packed bed. In the experimental work, a silica gel packed bed of eight layers has been studied. The objective of this work is to investigate the effect of the packed bed length on the bed performance for both adsorption and desorption processes.

Hamed et al. [6] studied experimentally the operation of an inclined bed of silica gel in adsorption and desorption modes of operation. This study considers only the mass transfer aspect to evaluate the effect of air velocity on the adsorption and desorption rates. The heat transfer and pressure drop are not considered in this study. It was found that heat and mass transfers' characteristics of the bed should therefore be 
adequately considered because of having important effects on the performance of the cooling system.

In very recent study, Chen et al. [7] investigated a silica gel circulating fluidized beds used for dehumidification of air conditioning system. In this study, air velocity varies between $4.0 \mathrm{~m} / \mathrm{s}$ and $6.0 \mathrm{~m} / \mathrm{s}$ and regeneration temperature varies between $40^{\circ} \mathrm{C}$ and $60^{\circ} \mathrm{C}$. This low temperature could be furnished by waste heat or solar energy. The results show that, increasing air velocity enhance dehumidification performance and increasing regeneration temperature enhance desorption performance.

Ramzi et al. [8] presented a mathematical model for heat and mass transfer in silica gel packed bed based on solid side resistance heat and consider conduction along the bed. Authors carried out adsorption and desorption experimental and numerical tests and examined the influence of diverse design parameters like air velocity, particle diameter, bed length and the number of units of mass transfer, on the significance of axial heat conduction. It has been found that the influence of axial heat conduction in the bed is reduced when increasing the particle diameter or increasing air flow velocity or diminishing the bed length. It is also proved that we must consider the axial heat conduction term when number of transfer units of mass and heat are superior to unity.

The objective of the present experimental study is to analyze heat and mass transfers and to investigate the effect of air flow rate and pressure drop on the bed performance for both adsorption and desorption processes. The experimental tests were carried out at nearly constant ambient parameters (humidity and temperature) and different values of air flow rate are used during adsorption and desorption processes.

\section{EXPERIMENTAL SETUP}

The experimental device (Figure 1) is composed of a centrifugal fan that circulates ambient air through a sheath in the test bed and then in the desiccator. A speed controller is used to change the air flow at the choice. Heating resistances are activated when studying regeneration phase.

The desiccator comprises two partitions: fixed and movable. The space between the two partitions is filled with desiccant (silica gel). Probes are used to measure temperature, speed and humidity and are placed at the entrance and the exit of the desiccator.

\subsection{Fan}

It is a centrifugal fan that circulates ambient air through a sheath in the test bed and then in the desiccator. The fan has a circular inlet and a square outlet. This fan blow air with an air flow up to $2000 \mathrm{~m}^{3} / \mathrm{h}$, with a power of $2200 \mathrm{~W}$, a voltage of $380 \mathrm{~V}$ and a pressure of $20 \mathrm{mmce}$.

\subsection{Variable speed drive}

A variable speed drive is a speed controller used to reduce or increase the flow air section, as needed, at the fan outlet. It creates a pressure drop causing air velocity and air flow change as wanted.

\subsection{Heating resistances}

Ten Heating resistances are composed of stainless steel fins. Nine resistances provide a power of $250 \mathrm{~W}$ each one of them. We use only the heating resistance that provides a power of $2 \mathrm{~kW}$. It is activated during regeneration phase in order to heat the air before it enters on the desiccator.

\subsection{The desiccator}

The desiccator is an iron and removable cylinder of $50 \mathrm{~cm}$ diameter. The desiccator is formed by fixed and movable partitions. The fixed partition is based on a perforated plate supporting the granular medium and ensuring the air circulation through the desiccator. The movable partition, with perforated base allowing air circulation, is guided by a rod and a lever. Then, it becomes easy to tighten the silica gel and to guide the partition.

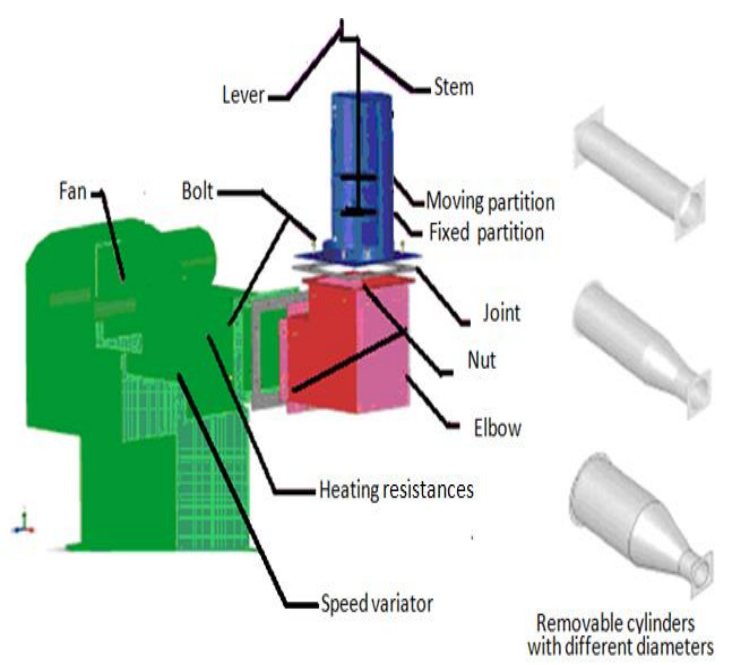

Figure 1. Mounting of the test bench drawn with 'solid works'

\subsection{Desiccant adsorbent}

We used silica gel as solid desiccant adsorbent. This used product can maintain the water vapor without being oozed or melted. It is white and non-toxic. The silica gel grains have an average grain diameter variable between 2 and $5 \mathrm{~mm}$.

\subsection{Measuring instruments}

The air velocity, temperature and humidity are measured by a portable device, KIMO VT300, with multi-function probes.

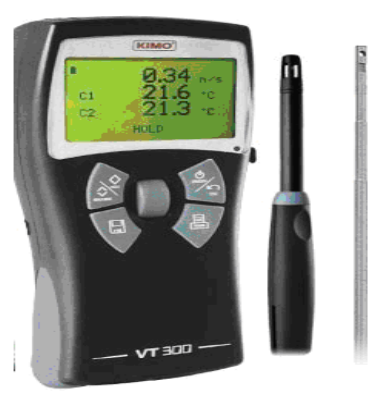

Figure 2-a. KIMO VT300 
1) The heat-hot-wire anemometer can measure different parameters: Speed, Automatic average point / Point Average, Average Point / Auto Focus, sheath and cones flow, PT100 temperature integrated in the probe.

2) The thermo-hygrometer can measure the following parameters: Relative humidity, absolute humidity, dew point, wet bulb temperature, Temperature PT100 integrated in the probe.

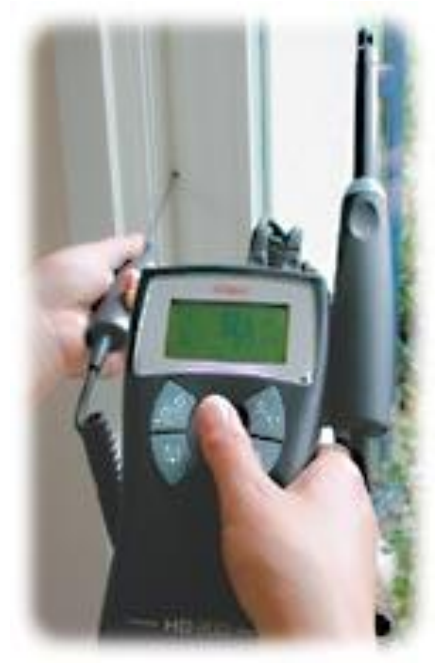

Figure 2-b. KIMO VT300 (how to use the KIMO)

The probes used are:

a) Hot wire probe

It is a probe of $8 \mathrm{~mm}$ of diameter and $300 \mathrm{~mm}$ of length (Figure 3). It measures speed and temperature ranging from 0 to $30 \mathrm{~m} / \mathrm{s}$ for velocity and from 20 to $80{ }^{\circ} \mathrm{C}$ for temperature. The resolution is about $0.01 \mathrm{~m} / \mathrm{s}$ when air velocity range from 0 to $3 \mathrm{~m} / \mathrm{s}$ and about $0.1 \mathrm{~m} / \mathrm{s}$ when velocity is beyond $3 \mathrm{~m} / \mathrm{s}$.

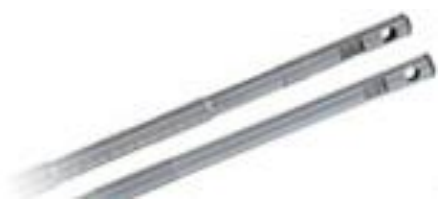

Figure 3. Hot wire probe

b) Humidity probe

It is a probe of $13 \mathrm{~mm}$ of diameter and $110 \mathrm{~mm}$ of length (Figure 4). It measures humidity as well as temperature. The measurement ranges are 3 to $98 \% \mathrm{RH}$ for moisture and from 20 to $+80{ }^{\circ} \mathrm{C}$ for temperature. The resolution is about $0.1{ }^{\circ} \mathrm{C}$ for the temperature and $0.1 \% \mathrm{RH}$ for moisture.

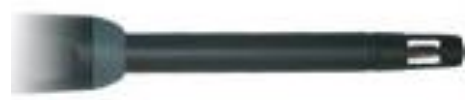

Figure 4. Humidity probe
The software DATA LOGGER 5.0 is used for configuration, save, visualization at real-time and exploitation of the Kimo probes measurements.

\section{RESULTS AND DISCUSSION}

Process air at nearly constant ambient parameters (humidity and temperature) and different values of air flow rate are used during adsorption and desorption processes.

The experimental results are obtained for different air flow value.

Table 1. Different experimental cases treated

\begin{tabular}{|l|l|l|l|l|l|l|}
\hline Case & $\begin{array}{l}\text { Diameter } \\
\mathrm{D}(\mathrm{cm})\end{array}$ & $\begin{array}{l}\text { Height } \\
\mathrm{H}(\mathrm{cm})\end{array}$ & $\begin{array}{l}\text { Velocity } \\
\mathrm{v}(\mathrm{m} / \mathrm{s})\end{array}$ & $\begin{array}{l}\text { Air flow } \\
\text { rate } \\
\mathrm{Qv}\left(\mathrm{m}^{3} / \mathrm{h}\right)\end{array}$ & $\begin{array}{l}\text { Volume } \\
\mathrm{V}\left(\mathrm{m}^{3}\right)\end{array}$ & $\begin{array}{l}\text { Aspect } \\
\text { ratio } \\
\mathrm{A}=\mathrm{R} / \mathrm{H}\end{array}$ \\
\hline 1 & 50 & 8 & 0.22 & 155.507 & 0.0157 & 3.125 \\
\hline 2 & 50 & 8 & 0.35 & 247.40 & 0.0157 & 3.125 \\
\hline 3 & 50 & 8 & 0.75 & 530.14 & 0.0157 & 3.125 \\
\hline
\end{tabular}

\subsection{Adsorption phase}

Figure 5-a represents the temporal evolution of air humidity and temperature at the desiccator outlet during the adsorption phase. At first, ventilated air observes his humidity decreasing. In fact, water vapor is adsorbed by the desiccant (silica gel). This dehumidification is accompanied by an undesirable increase in temperature. Then a temperature decrease occurs simultaneously with adsorption decrease. When silica gel becomes saturated, the adsorption phenomenon is inhibited and the moisture and temperature at reactor exit join their input values. Therefore, adsorption ensures a moisture reduction accompanied by an undesirable increase in temperature.

\subsection{Desorption phase}

After the adsorption phase, the desiccator needs to be regenerated which means that the used adsorbent must be dried once again for a further use. Solar capture is a possible device for regeneration. In this experience the needed heat is provided by heating resistances that raise the ambient air temperature.

Figure 5-b illustrates the temporal evolution of air humidity and temperature at the desiccator outlet during the desorption phase.

During desorption phase, hot air is ventilated over the desiccator resulting in silica gel temperature increase and causing evaporation. Therefore, a drop of water content in silica gel is denoted. The heated air crossing the saturated silica gel carries vapor with it resulting in elevation of exhausted air humidity and consequently temperature decreases. Gradually, as the silica gel is dried, air humidity moisture decreases and air temperature increases. After the regeneration process, silica gel is dried and air outlet temperature and humidity become similar to those at the desiccator inlet. 


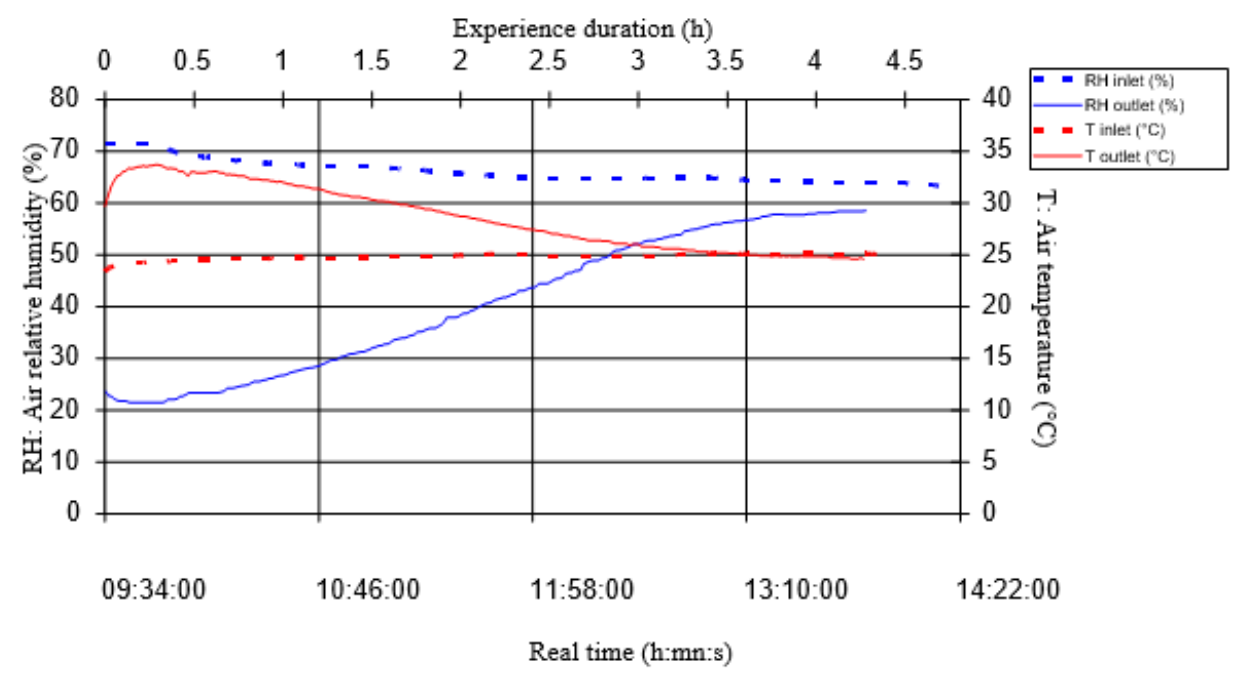

(a) Adsorption

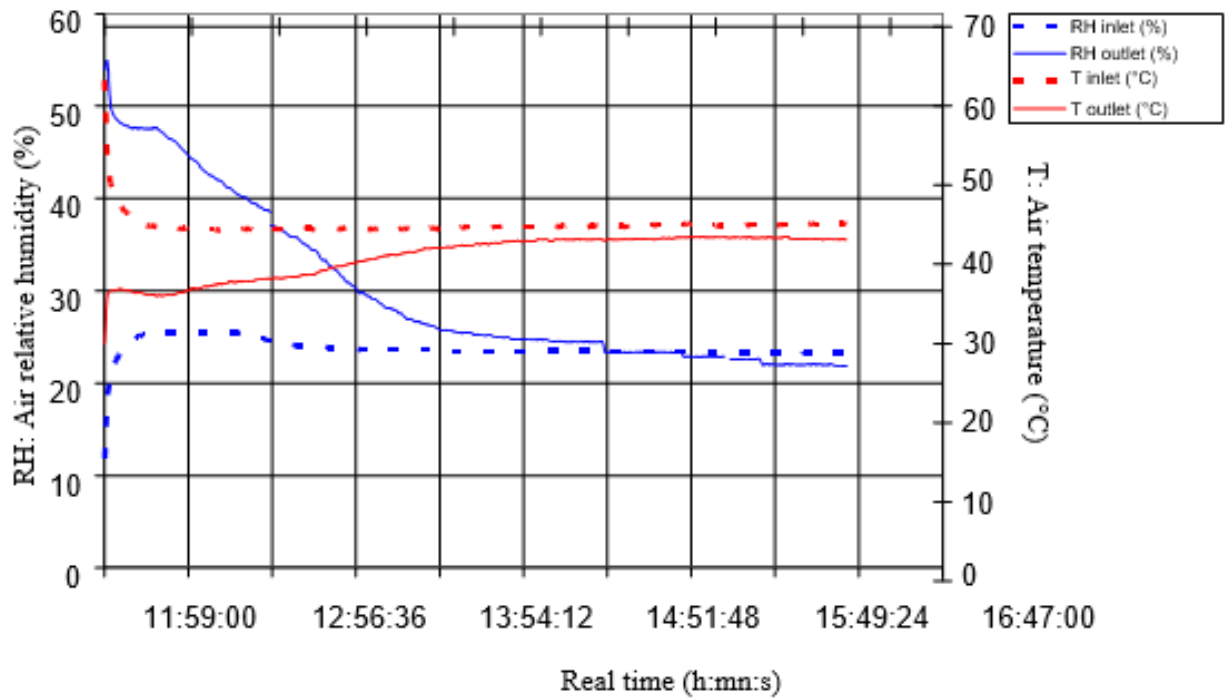

(b) Desorption

Figure 5. Temporal evolution of air relative humidity $(\mathrm{RH})$ and air temperature (T) during (a) adsorption phase and (b) desorption phase (Desiccator conditions: $\mathrm{Qv}=155.507 \mathrm{~m}^{3} / \mathrm{h}, \mathrm{V}=0.0157 \mathrm{~m}^{3}, \mathrm{D}=50 \mathrm{~cm}, \mathrm{H}=8 \mathrm{~cm}, \mathrm{v}=0.22 \mathrm{~m} / \mathrm{s}$ )

\subsection{Air flow rate effect}

a) During adsorption phase

Figure 6-a and Figure 6-b illustrate, respectively, effects of air flow rate on the temperature and humidity difference between the outlet and the inlet of the desiccators during adsorption phase. Humidity difference $\left(\mathrm{RH}_{\text {out }}-\mathrm{RH}_{\text {in }}\right)$ between the desiccator outlet $\left(\mathrm{RH}_{\text {out }}\right)$ and inlet $\left(\mathrm{RH}_{\text {in }}\right)$ (Table 2$)$ is more important for a lower air flow rate. In fact, when lowering the flow rate, the ambient air stays more in the reactor and loses more humidity. Consequently, its temperature increases further; temperature difference $\left(\mathrm{T}_{\text {out }}-\mathrm{T}_{\text {in }}\right)$ between the outlet $\left(\mathrm{T}_{\text {out }}\right)$ and the inlet $\left(\mathrm{T}_{\mathrm{in}}\right)$ of the desiccator is more important for a lower air flow rate.
Table 2. Air flow rate effect during adsorption phase

\begin{tabular}{|l|l|l|l|}
\hline $\begin{array}{l}\text { Velocity } \\
(\mathrm{m} / \mathrm{s})\end{array}$ & $\begin{array}{l}\text { Air flow rate } \\
\mathrm{Qv}\left(\mathrm{m}^{3} / \mathrm{h}\right)\end{array}$ & $\begin{array}{l}\left(\mathrm{RH}_{\text {out }}-\mathrm{RH}_{\text {in }}\right) \\
(\%)\end{array}$ & $\begin{array}{l}\left(\mathrm{T}_{\text {out }}-\mathrm{T}_{\text {in }}\right) \\
\left({ }^{\circ} \mathrm{C}\right)\end{array}$ \\
\hline 0.22 & 155.507 & 44.16 & 8.8 \\
\hline 0.35 & 247.40 & 34.43 & 6.5 \\
\hline 0.75 & 530.14 & 31 & 4.6 \\
\hline
\end{tabular}

We conclude the importance of having a low air flow rate during adsorption phase in order to promote the exchange between the silica gel and the treated air and thus results in an important air humidity reduction. 


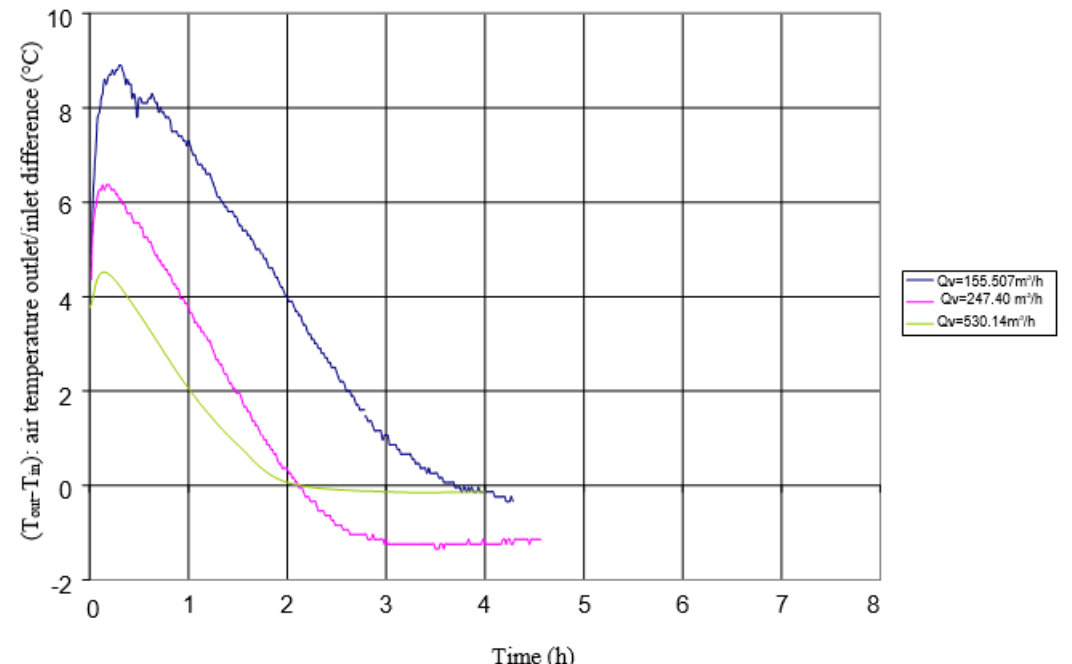

(a)
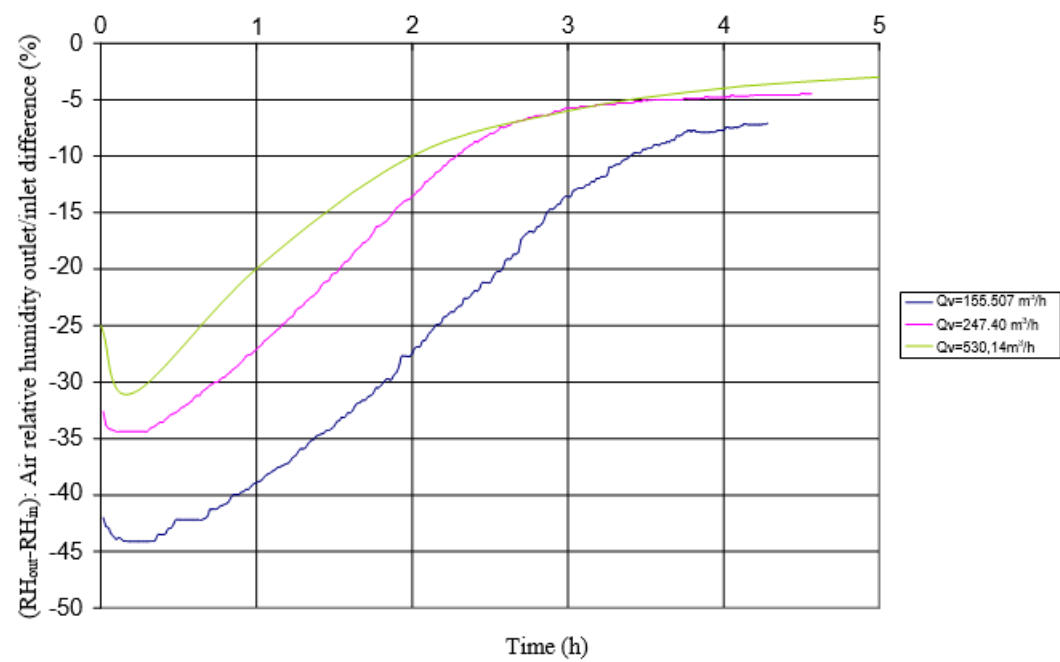

(b)

Figure 6. Effect of varying the flow rate on the temporal evolution of (a): $\left(T_{\text {out }}-T_{\text {in }}\right)$ : air temperature difference between outlet and inlet and (b): $\left(\mathrm{RH}_{\text {out }}-\mathrm{RH}_{\text {in }}\right)$ : air relative humidity difference between outlet and inlet during adsorption phase $\left(\mathrm{V}=0.0157 \mathrm{~m}^{3}\right.$ and $\mathrm{A}$ $=3.125$

b) During desorption phase

Figure 7-a and Figure 7-b illustrate, respectively, effects of air flow rate on the temperature and humidity differences between the outlet and the inlet of the desiccators during desorption phase.
As shown in Table 3, (the regeneration time is shorter for a higher air flow rate. The temperature and humidity stabilize more quickly (at the end of regeneration) for important air flow. So we conclude that the use of a high air flow rate during regenerating phase is recommended to accelerate this operation.

Table 3. Air flow rate effect during desorption phase

\begin{tabular}{|l|l|l|}
\hline $\begin{array}{l}\text { Velocity } \\
(\mathrm{m} / \mathrm{s})\end{array}$ & $\begin{array}{l}\text { Air flow rate } \\
\mathrm{Qv}\left(\mathrm{m}^{3} / \mathrm{h}\right)\end{array}$ & $\begin{array}{l}\text { Time duration of regeneration } \\
(\mathrm{h})\end{array}$ \\
\hline 0.75 & 530.14 & 1.55 \\
\hline 0.35 & 247.40 & 1.78 \\
\hline 0.22 & 155.507 & 3.7 \\
\hline
\end{tabular}




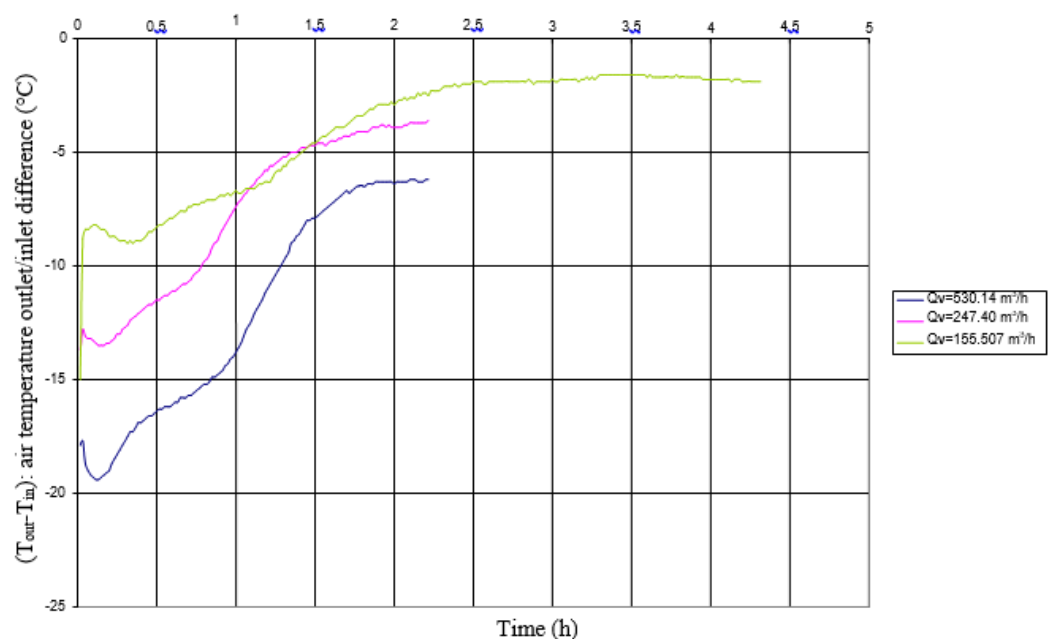

(a)

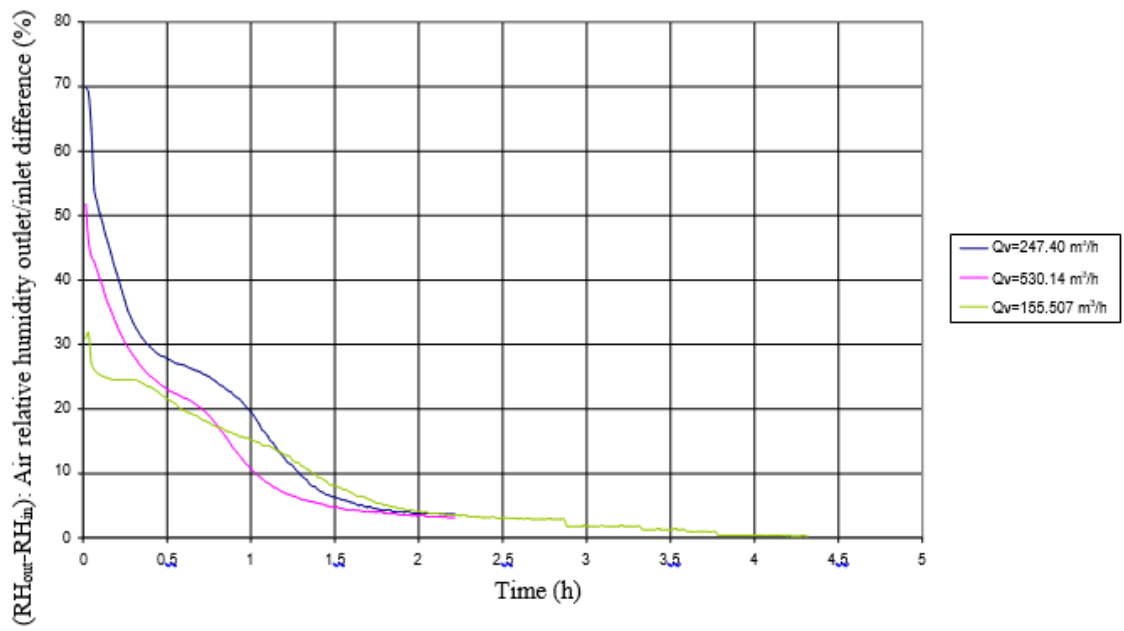

(b)

Figure 7. Effect of varying the flow rate on the temporal evolution of (a): $\left(T_{\text {out }}-T_{\text {in }}\right)$ : air temperature difference between outlet and inlet and (b): $\left(\mathrm{RH}_{\text {out }}-\mathrm{RH}_{\mathrm{in}}\right)$ : air relative humidity difference between outlet and inlet during desorption phase $\left(\mathrm{V}=0.0157 \mathrm{~m}^{3}\right.$ and $\mathrm{A}$ $=3.125)$

\subsection{Pressure drop}

The pressure drop in the desiccator is due to the wall roughness, the friction and the presence of silica gel bed, as a barrier to air flow, inside the cylinder. Thus, it is important to calculate the energy loss used in the desiccator design. The pressure drop in the studied medium was determined using a differential pressure gauge to measure pressure for different air flow rate.

Table 4. Pressure drop calculation for a cylinder of $\mathrm{D}=50 \mathrm{~cm}$ of diameter

\begin{tabular}{|l|l|l|l|}
\hline $\begin{array}{l}\text { Velocity } \\
\mathrm{v}(\mathrm{m} / \mathrm{s})\end{array}$ & 0.22 & 0.35 & 0.75 \\
\hline$\Delta \mathrm{P}(\mathrm{mbar})$ & 1 & 2 & 4 \\
\hline
\end{tabular}

Thus, we note that the pressure drop is more important for higher air flow rates. So, we deduce the importance of low air flow rate to reduce pressure drop.

\section{CONCLUSION}

In this study, the description of the experimental bed test and equipment is done. The obtained results were presented and analyzed. The aim was to analyze the adsorption and desorption as well as the effect of air flow rate on humidity and temperature temporal evolutions.

Experimental measurements indicate that the regeneration and adsorption rates are highly dependent on the air stream velocity.

From these results, we deduce that the use of low air flow rate through the desiccator is recommended to have important humidity reduction during the adsorption phase and to avoid pressure drop. We also conclude that the use of a high air flow rate during regenerating phase is needed to accelerate this operation. This information obtained from experimental studies allows us to optimize the desiccator design and thus facilitate the desiccant cooling system selection and design. 


\section{REFERENCES}

[1] "Development of solar desiccant humidifier," Research Manufacturing Company of California, Technical progress report, no. 87-14957-1, 1978.

[2] Dupont M., Celestine B., Nguyen P. H., Merigoux J. and Brandon B., "Desiccant solar air conditioning in tropical climates: I - dynamic experimental and numerical studies of silica gel and activated alumina," Solar Energy, vol. 52, pp. 509-17, 1994. DOI: 10.1016/0038-092X(94)90658-0.

[3] Hamed A. M., "Theoretical and experimental study on the transient adsorption characteristics of vertical packed porous bed," Renewable Energy, vol. 37, pp. 525-41, 2002. DOI: 10.1016/S0960-1481(01)00112-4.

[4] Kim S., Biswas P. and Mills A. F., "A compact low pressure drop desiccant bed for solar air conditioning applications, II bench scale tests," ASME, Journal of Solar Energy Engineering, vol. 107, pp. 120-127, 1985.
[5] Kabeel A. E., "Adsorption-desorption operations of multilayer desiccant packed bed for dehumidification applications," Renewable Energy, vol. 34, pp. 255265, 2009. DOI: $10.1016 /$ j.renene.2008.04.011.

[6] Ahmed M. Hamed, "Experimental investigation on the adsorption/desorption processes using solid desiccant in an inclined-fluidized bed," Renewable Energy, vol. 30, pp. 1913-1921, 2005. DOI: 10.1016/j.renene.2005.01.001.

[7] Chih-Hao Chen, Shu-Sheng Maa, Po-Hsun Wua Yuan-Ching Chiang and Sih-Li Chen, "Adsorption and desorption of silica gel circulating fluidized beds for air conditioning systems," App. Energy, vol. 155, pp. 708-718, 2015. DOI: 10.1016/j.apenergy.2015.06.041.

[8] Ramzy K. A., Kadoli R. and Ashok Babu T. P., "Significance of axial heat conduction in nonisothermal adsorption process in a desiccant packed bed," Int J Therm Sciences, vol. 76, pp. 68-81, 2014. 